\title{
One-Step Route to Synthesize Multiwalled Carbon Nanotubes Filled with MgO Nanorods
}

\author{
Jing Liu, ${ }^{1}$ Chunli Guo, ${ }^{2}$ Xiaojian Ma, ${ }^{1}$ Changhui Sun, ${ }^{1}$ Fengxia Li, ${ }^{1}$ and Yitai Qian ${ }^{1,3}$ \\ ${ }^{1}$ Key Laboratory of Colloid and Interface Chemistry, Shandong University, Ministry of Education, Jinan 250100, China \\ ${ }^{2}$ State Key Laboratory of Crystal Materials, Shandong University, Jinan 250100, China \\ ${ }^{3}$ Hefei National Laboratory for Physical Sciences at Microscale, Department of Chemistry, \\ University of Science and Technology of China, Anhui, Hefei 230026, China
}

Correspondence should be addressed to Chunli Guo, gcl@sdu.edu.cn and Yitai Qian, ytqian@ustc.edu.cn

Received 2 November 2009; Accepted 19 April 2010

Academic Editor: Yanqiu Zhu

Copyright ( 2010 Jing Liu et al. This is an open access article distributed under the Creative Commons Attribution License, which permits unrestricted use, distribution, and reproduction in any medium, provided the original work is properly cited.

Multiwalled carbon nanotubes filled with $\mathrm{MgO}$ nanorods were synthesized through the reaction of ethanol and $\mathrm{Mg}$ powder in the presence of $\mathrm{TiO}_{2}$ at $400^{\circ} \mathrm{C}$. X-ray powder diffraction indicated that the sample was composed of graphite and cubic $\mathrm{MgO}$. Transmission electron microscopy studies showed that multi-walled CNTs with the outer diameters of 70-130 nm were filled with discontinuous $\mathrm{MgO}$ nanorods whose diameter was in the range of $25-40 \mathrm{~nm}$. The ratios of the band intensities $\left(I_{D} / I_{G}=0.67\right)$ in Raman spectrum implied that carbon nanotubes had good crystallinity. The influence of correlative reaction factors on the morphology of the sample and the possible formation mechanism were discussed.

\section{Introduction}

Filling foreign materials into the hollow cavities of carbon nanotubes (CNTs) has been of widespread interest due to their special properties [1-3]. And they have broader application compared to pure CNTs or second phases, such as $\mathrm{Fe}_{2} \mathrm{O}_{3}$-filled CNTs as a negative electrode for an $\mathrm{Fe}$-air battery [4] and spherical $\mathrm{Ni}(\mathrm{OH})_{2} / \mathrm{CNTs}$ as the electrode in asymmetric supercapacitor [5]. $\mathrm{SnO}_{2} /$ multi-walled CNT has a high reversible discharge capacity compared to pure nano- $\mathrm{SnO}_{2}$ [6]. $\mathrm{MnO}_{2} / \mathrm{CNT}$ hybrid coaxial nanotubes have also enhanced reversible capacity more than pure $\mathrm{MnO}_{2}$ nanotubes [7].

$\mathrm{MgO}$ has gained extensive application in catalyst $[8,9]$, refractory materials [10] and optically transparent ceramic windows $[11,12]$. Recently the studies indicate that $\mathrm{MgO}$ can make ammoniated electrons stable [13] and has weak ferromagnetism [14]. Maybe the $\mathrm{MgO} / \mathrm{CNTs}$ have potential applications in electrode. And carbon-coated $\mathrm{MgO}$ nanoparticles had been prepared by various methods. Motiei et al. [15] had prepared hollow carbon cubes and carbon-coated $\mathrm{MgO}$ cubes through the reaction between $\mathrm{Mg}$ and $\mathrm{Mo}(\mathrm{CO})_{6}$ at $900^{\circ} \mathrm{C}$. Bedilo et al. [16] had synthesized carboncoated $\mathrm{MgO}$ nanoparticles by the decomposition of dry magnesium methoxide or resorcinol modified magnesium methoxide. Luo et al. [17] had synthesized $\mathrm{MgO} / \mathrm{C}$ coreshell nanospheres with the diameters of $300-500 \mathrm{~nm}$ at $600^{\circ} \mathrm{C}$ by reducing ethyl ether with metallic magnesium. Zhou et al. [18] had fabricated carbon-coated MgO by the coprecipitation magnesium nitrate with sucrose. However, there are few reports about the synthesis of CNT-coated MgO nanostructures.

In this study, we report the preparation and characterization of $\mathrm{MgO}$-filled multi-walled CNTs which were obtained by the reaction of ethanol and $\mathrm{Mg}$ powder in the presence of $\mathrm{TiO}_{2}$ at $400^{\circ} \mathrm{C}$. Transmission electron microscopy (TEM) showed that multi-walled CNTs filled with discontinuous $\mathrm{MgO}$ nanorods are open at the both ends, whose outer diameters were between 70 and $130 \mathrm{~nm}$. A possible mechanism for $\mathrm{MgO}$ nanorods encapsulated in multi-walled CNTs is discussed in this study and the research of electrochemical properties of $\mathrm{MgO} / \mathrm{CNT}$ is still underway.

\section{Experimental Section}

All reagents used in this work were analysis-grade reagents from the Shanghai Chemical Factory, China, and were 
directly used without further treatment. $\mathrm{MgO}$ nanorods encapsulated in multi-walled CNTs were prepared as follows: magnesium powder $(1 \mathrm{~g})$, ethanol $(10 \mathrm{~mL})$, and $\mathrm{TiO}_{2}(0.5 \mathrm{~g})$ were mixed in a $20 \mathrm{~mL}$ stainless autoclave and heated in an electric oven at $400^{\circ} \mathrm{C}$ for 10 hours, then cooled to room temperature. The wet black powder was collected and washed with dilute hydrochloric and hydrofluoric. The final products were washed with distilled water and absolute ethyl alcohol, and then dried under vacuum at $50^{\circ} \mathrm{C}$ for 4 hours.

X-ray powder diffraction (XRD) measurements were carried out using a Bruker D8 advanced X-ray diffractometer equipped with graphite-monochromatized $\mathrm{Cu} \mathrm{K} \alpha$ radiation $(\lambda=1.5418 \AA)$. Field-emission scanning electron microscopy (FESEM) images were taken by a JSM-6700F scanning electron microscope. TEM images were obtained by a Hitachi model H-7000 TEM with an accelerating voltage of $100 \mathrm{kV}$. The high-resolution (HRTEM) images and energy-dispersive X-ray spectroscopy (EDS) were recorded on a JEOL 2100 HRTEM microscope. Raman spectra were obtained using a Horiba Jobin Yvon Raman microscope system. The spectrometer was operated in continuous scanning mode in the range of $500-1700 \mathrm{~cm}^{-1}$ using argon-ion laser of wavelength $\lambda=514.5 \mathrm{~nm}$.

\section{Results and Discussion}

Figure 1 shows XRD pattern of the as-prepared products. The diffraction peaks at 43.0 and $62.4^{\circ}$ were identified as the (200) and (220) reflections of MgO (JCPDS card no. 65-0476), and those at 26.5 and $44.9^{\circ}$ were attributed to the (002) and (101) planes of CNT-related graphite (marked with " $\mathrm{c}$ "). There are $\mathrm{TiC}$ and a little stable rutile-type titanium dioxide [19] in the XRD pattern.

Figure 2(a) is a SEM image of the samples washed with $\mathrm{HCl}$. A great number of CNTs with outer diameters of $70 \sim 130 \mathrm{~nm}$ and lengths of tens of micrometers were observed in Figure 2(a). The top of nanotube displayed in the inset of Figure 2(a) is open, which likes nanocapillary to absorb hydrogen or other gases [20]. Figure $2(\mathrm{~b})$ is a TEM image of the samples. The internal structure of CNTs, inside which discontinuous nanorods were encapsulated with the diameters of $25-40 \mathrm{~nm}$, was observed clearly. The inset in Figure 2(b) is the corresponding EDS of nanorods, suggesting that their chemical compositions were $\mathrm{MgO}$ (the $\mathrm{Cu}$ peaks are from the copper grid that supports the specimen and $\mathrm{C}$ peaks are possibly from the nanotubes). That Ti peak was not observed in EDS indicated that TiC particles were not located inside CNTs. The TEM image of the aggregated particles located outside CNTs was further confirmed (see Figure S1 in supplementary material available online at doi:10.1155/2010/671863 supporting information). A further investigation by HRTEM (Figure 2(c)) reveals that the interlayer separation of $0.34 \mathrm{~nm}$ corresponds to the (002) plane of the multi-walled CNTs and the fringe spacing of $0.21 \mathrm{~nm}$ matches the (200) reflection of $\mathrm{MgO}$ nanorods.

The representative Raman spectrum (Figure 3) of the sample shows the typical features of multi-walled CNTs.

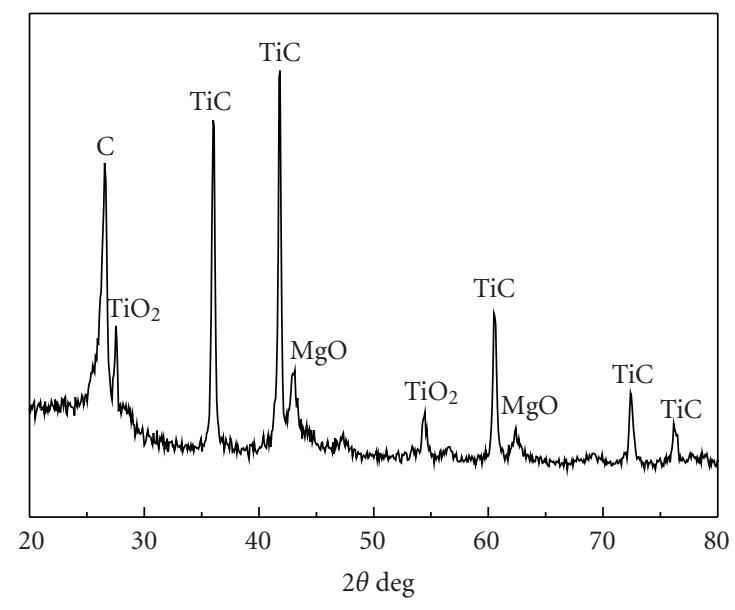

FIGURE 1: The typical XRD patterns of the products washed with dilute acid.

The spectrum demonstrates that the peak frequencies of the graphite $(\mathrm{G})$ mode are at $1575 \mathrm{~cm}^{-1}$ and contains disorder (D) modes that are at $1343 \mathrm{~cm}^{-1}$. An indication of the relative graphitization of the deposited material can be expressed as the ratio of $I_{D} / I_{G}$ [21]. The ratios of the band intensities shown in Figure 3 are $I_{D} / I_{G}=0.67$, suggesting a higher degree of graphitization.

Several experimental factors including metal oxides, temperature, and metals were adopted to investigate the possible formation mechanism of the MgO-filled multiwalled CNTs. The introduction of metal oxides played a key role in encapsulating $\mathrm{MgO}$ nanorods inside multi-walled CNTs. In the absence of $\mathrm{TiO}_{2}$, only pure multi-walled CNTs appeared in the products, which agreed with previous results [22]. If the amount of $\mathrm{TiO}_{2}$ was less than $0.5 \mathrm{~g}$ in our experimental route, the encapsulations of $\mathrm{MgO}$ inside multiwalled CNTs were difficult to be observed by TEM. As $\mathrm{TiO}_{2}$ was replaced by other metal oxides such as $\mathrm{Fe}_{2} \mathrm{O}_{3}$, the main morphology of as-prepared samples was different with that of $\mathrm{TiO}_{2}$ added in the reaction system. The abovementioned experimental results suggested that the presence of $\mathrm{TiO}_{2}$ in the reaction system could assist the formation of $\mathrm{MgO}$ nanorods encapsulated in multi-walled CNTs. The detail reason for the influence of metal oxides on the products was still under investigation. In addition, the influences of the temperature and metals on the $\mathrm{MgO}$-filled multi-walled CNTs had also been studied. Results showed that the yield of $\mathrm{MgO}$ nanorods encapsulated inside multi-walled CNTs was reduced with the reaction temperature decreasing. When the reaction temperature was lower than $200^{\circ} \mathrm{C}$, irregular graphite films were the major products. Furthermore, when $\mathrm{Mg}$ powder was substituted by $\mathrm{Al}, \mathrm{Fe}, \mathrm{Zn}$, $\mathrm{Co}$, or $\mathrm{Ni}$, the reaction did not happen. A possible reason is that the activity of $\mathrm{Mg}$ powder was higher than that of abovementioned metals.

Based on the results of HRTEM and EDS, a possible growth mechanism of multi-walled CNTs filled with $\mathrm{MgO}$ nanorods was proposed. It is known that catalyst materials 


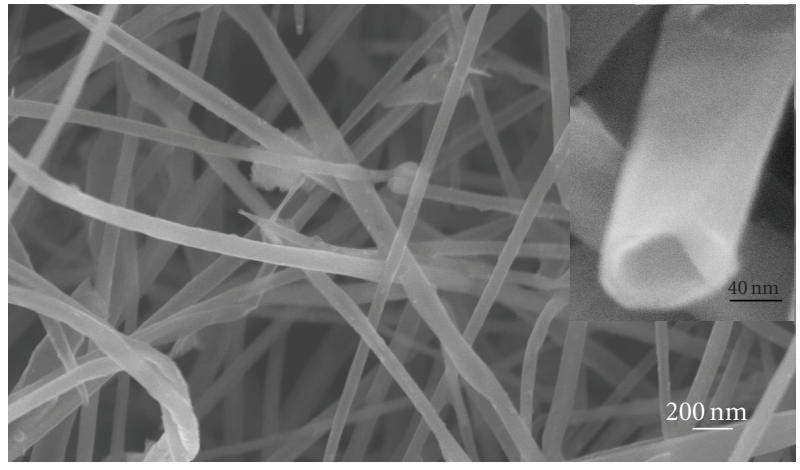

(a)

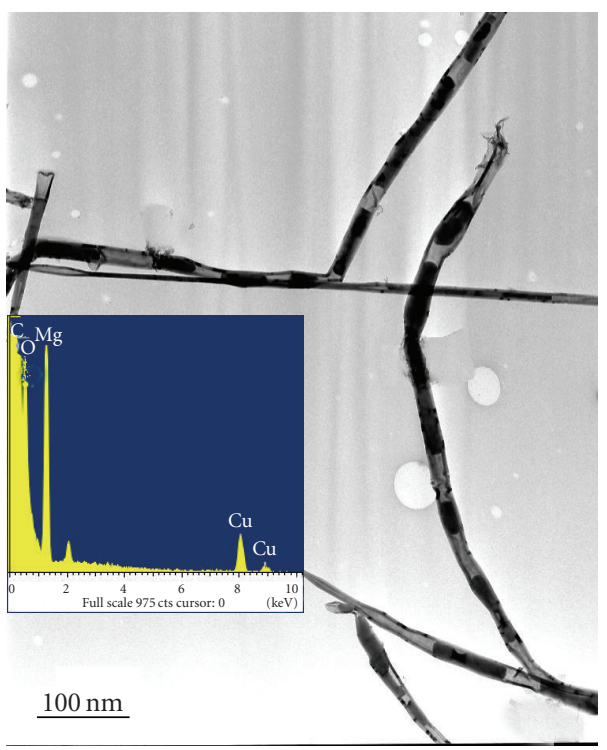

(b)

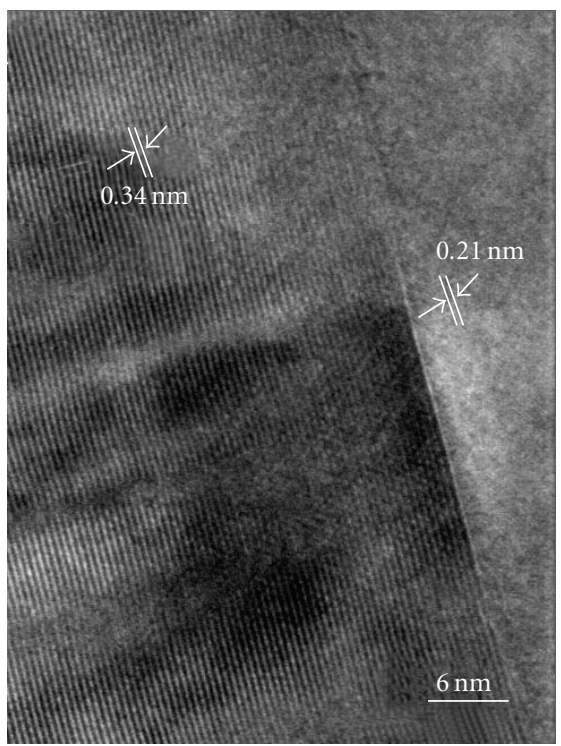

(c)

FIgure 2: (a) SEM image of CNTs (The inset is a high-resolution image.); (b) TEM image of CNTs filled with MgO nanorods (inset, EDS); (c) HRTEM image of multi-walled CNTs filled with MgO.

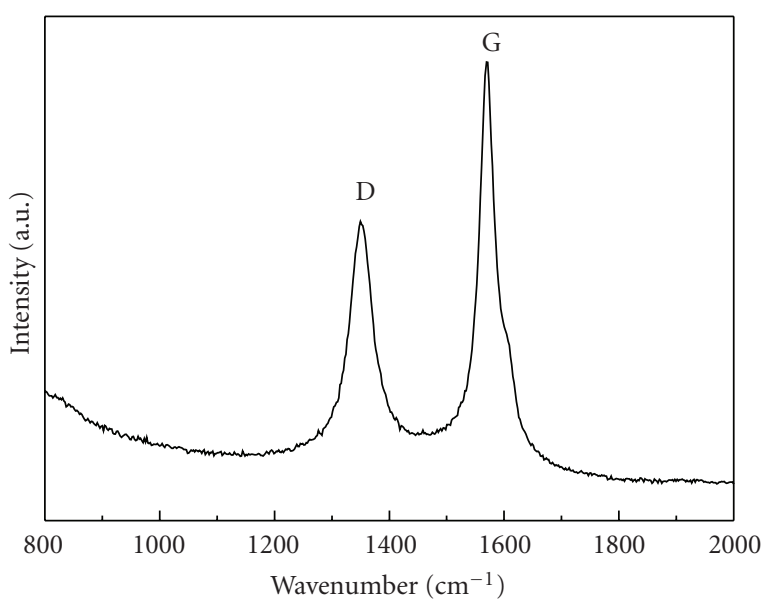

FIGURE 3: Raman spectrum of the as-prepared sample.

at the ends of CNTs were easy to be encapsulated into the CNTs [23-25]. However, in this system, there were no magnetic catalysts to promote the encapsulation and their growth mechanism cannot be used to explain the growth of the MgO-filled multi-walled CNTs. And there are no other reports on the encapsulation of $\mathrm{MgO}$ nanostructures inside multi-walled CNTs. Some porous $\mathrm{MgO}$ nanorods with the diameters of $25-40 \mathrm{~nm}$, similar in size to the $\mathrm{MgO}$ nanorods encapsulated by multi-walled CNTs, were observed by TEM (Figure 4(a)). The inset in Figure 4(a) is an enlarged individual porous $\mathrm{MgO}$ nanorod, which was characterized by HRTEM (Figure 4(b)). The formation of $\mathrm{MgO}$ may be ascribed to the reaction of $\mathrm{Mg}$ power and $\mathrm{TiO}_{2}$. Simultaneously TiC nanoparticles form between Ti coming from $\mathrm{TiO}_{2}$ and carbon atoms. The inset in Figure 4(b) further proved that the porous nanorods were coated with layers of graphite. Porous structures have high specific surface area and high surface energy [26]. Therefore carbon atoms were easy to deposit on the surface of the $\mathrm{MgO}$ nanorods. As the reaction proceeded, the graphite layers turned into highly ordered multi-walled CNTs and finally the MgO-filled CNTs were formed. 


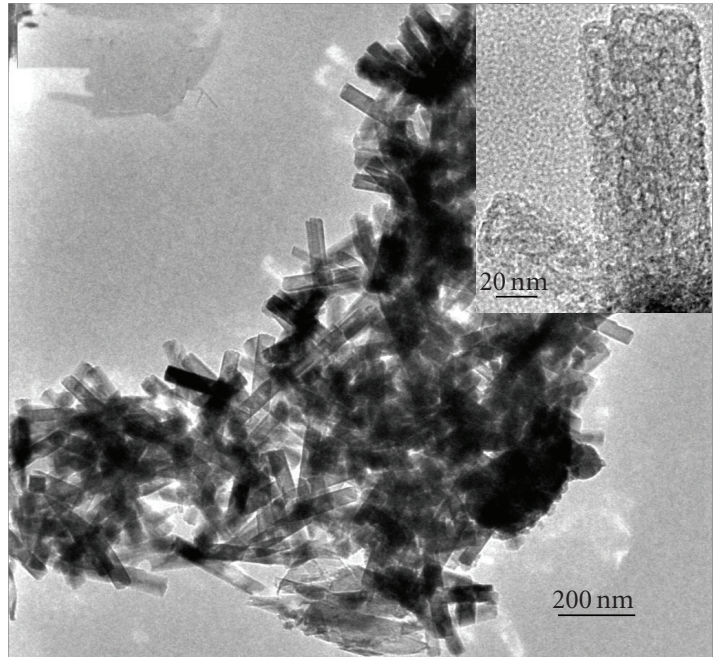

(a)

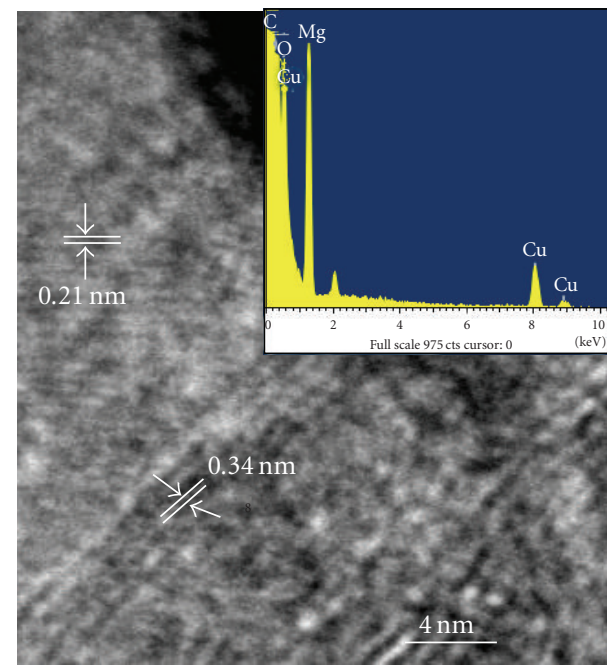

(b)

FIGURE 4: (a) TEM image of the porous products (The inset image shows the enlarged nanorods.); (b) HRTEM analysis of the porous $\mathrm{MgO}$ nanorod (The inset image is the EDS spectrum.).

\section{Conclusions}

$\mathrm{MgO}$ nanorods encapsulated in multi-walled CNTs were fabricated by a one-step synthesis in a $20 \mathrm{~mL}$ autoclave at $400^{\circ} \mathrm{C}$. TEM and HRTEM images show that $\mathrm{MgO}$ nanorods with diameters of 25-40 nm were encapsulated inside multiwalled CNTs with outer diameters of 70-130 nm and lengths of tens of micrometers. Raman spectrum suggested that multi-walled CNTs had excellent crystalline. The $\mathrm{MgO}$ nanorods are believed to be a template for the multiwalled CNTs growing. The electrochemical properties of $\mathrm{MgO} / \mathrm{CNT}$ are being studied.

\section{Acknowledgments}

Financial supports from the National Natural Science Foundation of China (no. 20671058), the 973 Projects of China (no. 2005CB623601), and the Natural Science Foundation of Shandong province (no. 11190004010664) are greatly appreciated. The authors thank Dr. Pamela Holt, Shandong University, for proofreading the manuscript.

\section{References}

[1] P. M. Ajayan and S. Iijima, "Capillarity-induced filling of carbon nanotubes," Nature, vol. 361, no. 6410, pp. 333-334, 1993.

[2] D. Ugarte, A. Châtelain, and W. A. de Heer, "Nanocapillarity and chemistry in carbon nanotubes," Science, vol. 274, no. 5294, pp. 1897-1899, 1996.

[3] J. Sloan, J. Cook, M. L. H. Green, J. L. Hutchison, and R. Tenne, "Crystallisation inside fullerene related structures," Journal of Materials Chemistry, vol. 7, no. 7, pp. 1089-1095, 1997.

[4] B. T. Hang, H. Hayashi, S.-H. Yoon, S. Okada, and J.-I. Yamaki, " $\mathrm{Fe}_{2} \mathrm{O}_{3}$-filled carbon nanotubes as a negative electrode for an Fe-air battery," Journal of Power Sources, vol. 178, no. 1, pp. 393-401, 2008.

[5] X.-F. Wang, D.-B. Ruan, and Z. You, "Application of spherical $\mathrm{Ni}(\mathrm{OH})_{2} / \mathrm{CNTs}$ composite electrode in asymmetric supercapacitor," Transactions of Nonferrous Metals Society of China, vol. 16, no. 5, pp. 1129-1134, 2006.

[6] Y. Fu, R. Ma, Y. Shu, Z. Cao, and X. Ma, "Preparation and characterization of $\mathrm{SnO}_{2}$ /carbon nanotube composite for lithium ion battery applications," Materials Letters, vol. 63, no. 22, pp. 1946-1948, 2009.

[7] A. L. M. Reddy, M. M. Shaijumon, S. R. Gowda, and P. M. Ajayan, "Coaxial $\mathrm{MnO}_{2}$ /carbon nanotube array electrodes for high-performance lithium batteries," Nano Letters, vol. 9, no. 3, pp. 1002-1006, 2009.

[8] D. Gulková, O. Šolcová, and M. Zdražil, "Preparation of MgO catalytic support in shaped mesoporous high surface area form," Microporous and Mesoporous Materials, vol. 76, no. 1-3, pp. 137-149, 2004.

[9] M. J. Climent, A. Corma, S. Iborra, and M. Mifsud, "MgO nanoparticle-based multifunctional catalysts in the cascade reaction allows the green synthesis of anti-inflammatory agents," Journal of Catalysis, vol. 247, no. 2, pp. 223-230, 2007.

[10] M.-A. Faghihi-Sani and A. Yamaguchi, "Oxidation kinetics of MgO-C refractory bricks," Ceramics International, vol. 28, no. 8, pp. 835-839, 2002.

[11] R. Chaim, Z. Shen, and M. Nygren, "Transparent nanocrystalline $\mathrm{MgO}$ by rapid and low-temperature spark plasma sintering," Journal of Materials Research, vol. 19, no. 9, pp. 2527-2531, 2004.

[12] D. Chen, E. H. Jordan, and M. Gell, "Pressureless sintering of translucent MgO ceramics," Scripta Materialia, vol. 59, no. 7, pp. 757-759, 2008.

[13] M. Chiesa, E. Giamello, and S. Van Doorslaer, "Ammoniated electrons stabilized at the surface of MgO," Journal of the American Chemical Society, vol. 131, no. 35, pp. 12664-12670, 2009.

[14] J. I. Beltrán, C. Monty, Ll. Balcells, and C. Martínez-Boubeta, "Possible d0 ferromagnetism in MgO," Solid State Communications, vol. 149, no. 39-40, pp. 1654-1657, 2009.

[15] M. Motiei, J. Calderon-Moreno, and A. Gedanken, "The formation of carbon-coated $\mathrm{MgO}$ cubes and carbon cubes," Advanced Materials, vol. 14, no. 16, pp. 1169-1172, 2002.

[16] A. F. Bedilo, M. J. Sigel, O. B. Koper, M. S. Melgunov, and K. J. Klabunde, "Synthesis of carbon-coated MgO nanoparticles," Journal of Materials Chemistry, vol. 12, no. 12, pp. 3599-3604, 2002. 
[17] T. Luo, X. Yang, J. Liu, W. Yu, and Y. Qian, "A core-shell nanostructure of carbon," Chemistry Letters, vol. 34, no. 2, pp. 168-169, 2005.

[18] Q. Zhou, J.-W. Yang, Y.-Z. Wang, Y.-H. Wu, and D.-Z. Wang, "Preparation of nano-MgO/Carbon composites from sucroseassisted synthesis for highly efficient dehydrochlorination process," Materials Letters, vol. 62, no. 12-13, pp. 1887-1889, 2008.

[19] M. Kobayashi, V. Petrykin, M. Kakihana, and K. Tomita, "Hydrothermal synthesis and photocatalytic activity of whisker-like rutile-type titanium dioxide," Journal of the American Ceramic Society, vol. 92, no. s1, pp. S21-S26, 2009.

[20] C. Bower, W. Zhu, D. Shalom, D. Lopez, L. H. Chen, P. L. Gammel, and S. Jin, "Large current density from carbon nanotube field emitters," Applied Physics Letters, vol. 80, p. 3820, 2002.

[21] W. Huang, Y. Wang, G. Luo, and F. Wei, "99.9\% purity multi-walled carbon nanotubes by vacuum high-temperature annealing," Carbon, vol. 41, no. 13, pp. 2585-2590, 2003.

[22] J. Liu, M. Shao, X. Chen, W. Yu, X. Liu, and Y. Qian, "Largescale synthesis of carbon nanotubes by an ethanol thermal reduction process," Journal of the American Chemical Society, vol. 125, no. 27, pp. 8088-8089, 2003.

[23] V. Jourdain, H. Kanzow, M. Castignolles, A. Loiseau, and P. Bernier, "Sequential catalytic growth of carbon nanotubes," Chemical Physics Letters, vol. 364, no. 1-2, pp. 27-33, 2002.

[24] C. Ducati, I. Alexandrou, M. Chhowalla, G. A. J. Amaratunga, and J. Robertson, "Temperature selective growth of carbon nanotubes by chemical vapor deposition," Journal of Applied Physics, vol. 92, no. 6, pp. 3299-3306, 2002.

[25] Y. Li, J. Chen, Y. Ma, J. Zhao, Y. Qin, and L. Chang, "Formation of bamboo-like nanocarbon and evidence for the quasi-liquid state of nanosized metal particles at moderate temperatures," Chemical Communications, no. 12, pp. 1141-1142, 1999.

[26] M. H. Lee and D. G. Park, "Preparation of MgO with high surface area, and modification of its pore characteristics," Bulletin of the Korean Chemical Society, vol. 24, no. 10, pp. 1437-1443, 2003. 

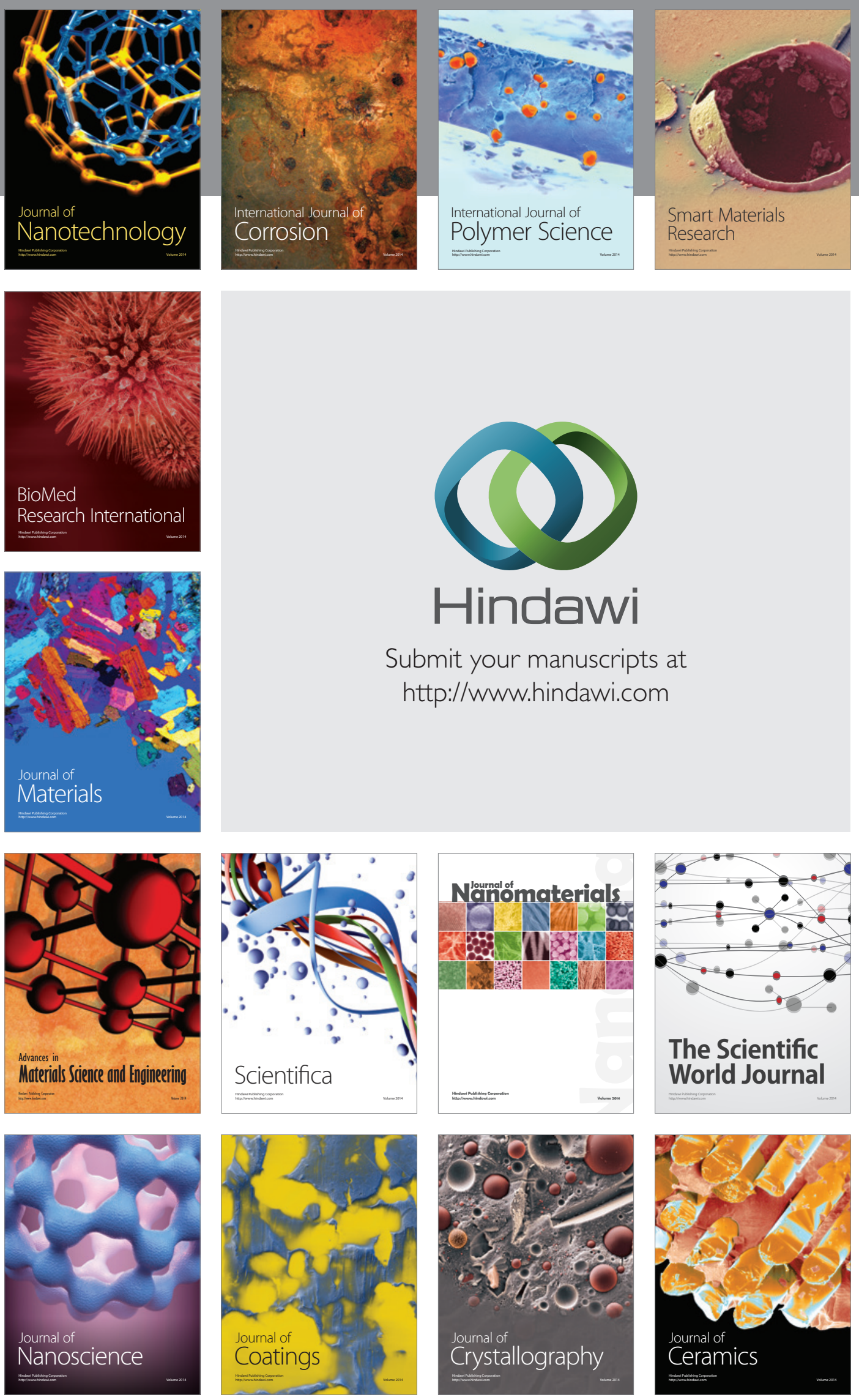

The Scientific World Journal

Submit your manuscripts at

http://www.hindawi.com

\section{World Journal}

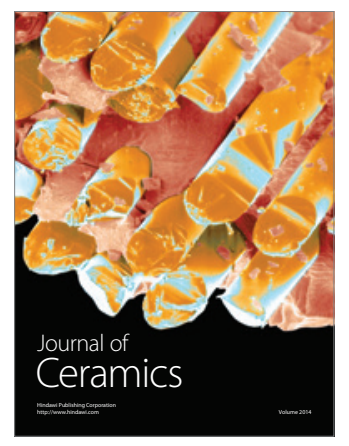

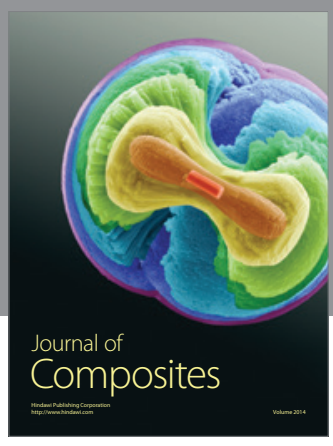
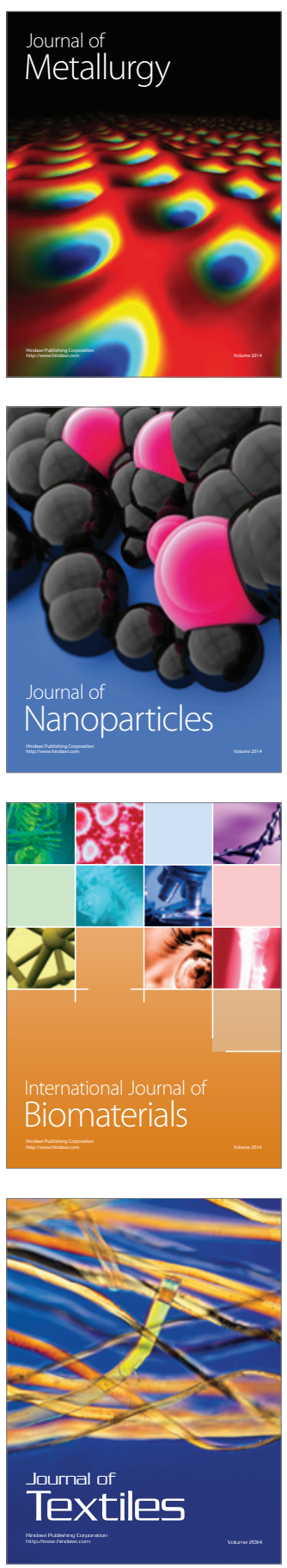